\title{
Wavelength switching and light modulation in laser diodes with nonidentical multiple quantum wells
}

\author{
${ }^{\mathrm{a}}$ Yi-Shin Su, ${ }^{\mathrm{a}}$ Chao-Hsin Wu and ${ }^{\mathrm{a}, \mathrm{b}}$ Ching-Fuh Lin \\ a with Graduate Inst. of Electron-Optical Engr., National Taiwan Univ., Taiwan \\ ${ }^{b}$ with Dept. of Electrical Engr.and Graduate Inst. of Electronics Engr., National Taiwan Univ., \\ Taiwan
}

\begin{abstract}
The wavelength of the designed two-section laser diode can be modulated by direct current modulation. The modulation speed of intensity of each wavelength is 1.5 times faster than the speed of a direct modulated single section laser. The modulation depth of output intensity can be $20 \mathrm{~dB}$.
\end{abstract}

Keywords: laser diode, two section, two wavelength, wavelength switching, intensity modulation

\section{INTRODUCTION}

A communication system is composed of receivers, signal path, and transmitters. Using light as the signal carrier is advantagous over using electrical signals or RF signals because the light signals suffer less distortion and loss [1][2]. Thus, the bit rate can be higher and communication distance can be longer. In addition, different channels can use the same signal path because signals carried by different wavelengths do not interact with each other [3]. To fully take the advantage of light, high-speed and broad wavelength bandwidth receivers and transmitters are required. Detectors with $40 \mathrm{GHz}$ bandwidth are developed early and are commercially available. Detectors with more than $100 \mathrm{GHz}$ bandwidth are under development and will be commercially available, soon. However, the speed and wavelength bandwidth of light sources available is far below the requirement. The only $40 \mathrm{GHz}$ transmitters commercially available now are assembled from CW light sources and light modulators [4][5][6]. The wavelength bandwidth of these systems are mostly below $30 \mathrm{~nm}$ because of the difficulty to integrate wavelength tuning elements on laser diodes [7]. In addition, these transmitters have higher production cost, require more space and more power. Thus, the research efforts to increase the speed of direct modulated laser diodes are never stopped. Currently, the speed of commercially available lasers is about $10 \mathrm{~Gb} / \mathrm{s}$. Laser diodes with $22 \mathrm{GHz}$ modulation bandwidth is developed on quantum dot structures because it has the unique delta function like density of states and high differential gain [8]. The fastest laser diode developed to date, which has $43 \mathrm{GHz}$ bandwidth, is the tunneling injection quantum well laser because the carriers can flow in and out quickly by tunneling [9]. For applications that requires more than $43 \mathrm{GHz}$ bandwidth, there is no suitable direct modulated light sources. Here we propose a new type of laser design in which the laser wavelength can be switched by changing the injection current. In addition, the intensity modulation of the laser oscillations can be faster than normal quantum well lasers for its unique wavelength switching mechanism.

\section{DEVICE DESIGN AND FABRICATION}

The device proposed is a Fabry-Perot Laser diode with two-section waveguide fabricated on an InP substrate. The active layer consists of two quantum wells designed for $1.5 \mu \mathrm{m}$ wavelength emission and three quantum wells designed for $1.3 \mu \mathrm{m}$ wavelength emission. The two types of quantum wells are inserted between each other as shown in the following figure. 


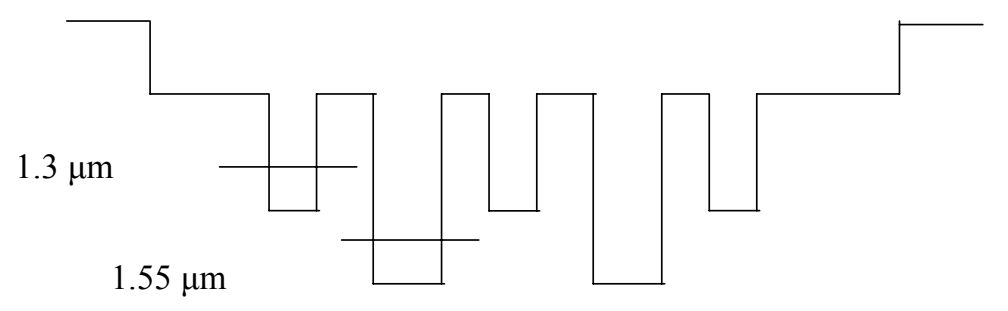

Figure 1: The active layer structure.

The devices use double channel ridge waveguides. The waveguide is $3 \mu \mathrm{m}$ in width. The channel is etched by RIE. The process gas is $\mathrm{CH}_{4}$ and $\mathrm{H}_{2}$. The etched depth is 100 200nm above the undoped optical confinement layer. As shown in Figure 2, the gap between two waveguide sections is also etched during waveguide etching process. Thus, the p-cladding of the two sections are electrically isolated. The optical confinement layer is not etched. Thus, the light passing from one section to the other will not be reflected by air-semiconductor interface.

After the waveguide is created by dry-etching, $3 \mathrm{k} \AA$ PECVD oxide is applied as insulation layer. The oxide grown on top of the waveguide is removed by self align technique. The p-side contact is Ti/Pt/Au deposited by an e-beam evaporator. The thickness is $0.5 \mathrm{k} \AA / 0.75 \mathrm{k} \AA / 4.5 \mathrm{k} \AA$. For devices used in frequency response measurement, the Ti/Pt/Au bonding pad is reduced to $70 \mu \mathrm{m} \times 70 \mu \mathrm{m}$ in order to reduce the parasitic capacitance of the $\mathrm{p}$-metal/oxide/semiconductor three-layer structure. Finally, the wafer is lapped to $100 \mu \mathrm{m}$ thick and cleaved into devices. As shown in Figure 3, the cleaved device is $1 \mathrm{~mm}$ in length. It has two sections of $500 \mu \mathrm{m}$ waveguides.

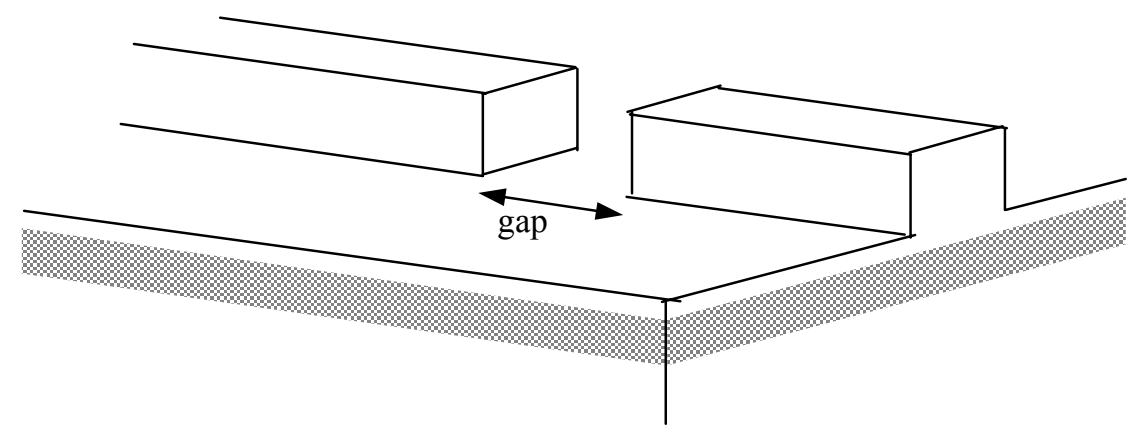

Figure 2 : The etched gap between two waveguide sections. The red area is the active region.

I1

I2

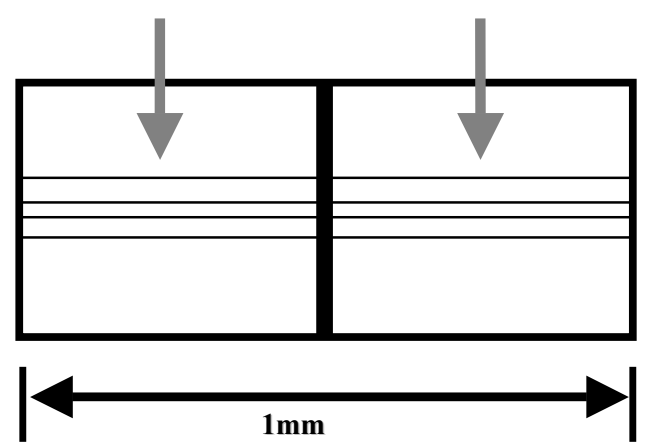

Figure 3 : The fabricated two section laser diode. 


\section{THE MODEL OF WAVELENGTH SWITCHING}

Because the gain profile of the designed quantum well varies with the injected current density [10], the combined gain profile will change as the relative injected current density changes. As shown in Figure 4, the dot line is the gain contributed by I 2 and dash-dot line the gain contributed by I1, assuming I 2 is larger than I1. The gain contributed by I2 is peaked at $\lambda 2$. When I 2 is increased, the gain contributed by I 2 to both $\lambda 1$ and $\lambda 2$ will increase. However, the increase of gain at $\lambda 2$ will be more than the increase of gain at $\lambda 1$ because the gain contributed by I 2 to $\lambda 1$ is saturated due to the band-filling effect. Thus, the laser oscillation at $\lambda 2$ will increase. In addition, the increased oscillation at $\lambda 2$ will suppress the carrier population contributed by I1, and the gain contributed by I1 to $\lambda 1$ will decrease, leading to decrease of laser oscillation intensity at $\lambda 1$. When I1 is increased, the gain contributed by I1 to both $\lambda 1$ and $\lambda 2$ will increase, too. However, because of the lower injection level of I1, band-filling effect does not dominate. The increased carriers will populate lower states and contribute more gain to $\lambda 1$ than to $\lambda 2$. As a result, the laser oscillation at $\lambda 1$ will increase. Also, the increased laser oscillation at $\lambda 1$ will suppress the population contributed by I 2 and leads to the decrease of laser oscillations at $\lambda 2$.

During the switching process, the changes of carrier populations in the two sections have different reasons. For instance, when I1 is increased, the carriers in section 1 are increased due to increase of I1. This will lead to the increase of laser intensity at $\lambda 1$. Then, laser oscillations at $\lambda 1$ will compete with laser oscillations at $\lambda 2$ for carriers contributed by I2, resulting in carriers, which originally populate at higher energy levels, fall to lower energy levels. Hence the increased oscillation intensity at $\lambda 1$ has two sources of contribution. One is the increased current, I1. The other is the carrier, which are initially at higher energy states. These carries fall to lower states because of gain competition and so contribute optical gain to $\lambda 1$. The transition time between any two states in the active layer is much less than the time required for carrier to travel from the electrical contact layer to the active layer. Thus, the carrier population should be able to reach equilibrium faster than a single section laser diode, which does not have two oscillation wavelengths.

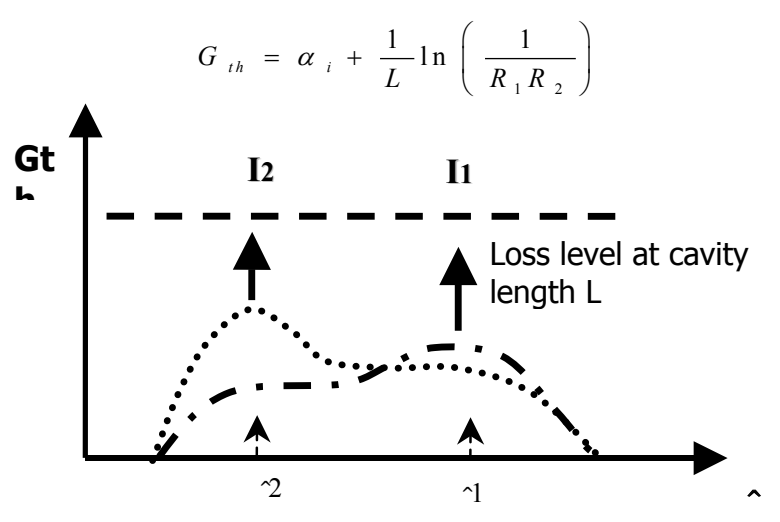

Figure 4: By controlling I1 and i2, the shape of total gain spectrum can be changed, leading to change of oscillation wavelength.

\section{STEADY STATE RESPONCE}

For steady state response measurement, the device used has $10 \mu \mathrm{m}$ gap. It is mounted on a copper heat sink and a gold wire is bonded on each contact by indium solder as shown in Figure 5. To reduce the effect of heating, two HP8114A pulse source is used as current source for this device. The output pulses have $1 \mathrm{kHz}$ repetition rate and $1 \mu$ s width. The output from one facet is collimated to a multimode fiber and sent to an optical spectrum analyzer. From another facet, the two groups of laser oscillations are separated by a grating and collected by two detectors as shown in Figure 6. In Figure 6 , the symbols "I1" and "I2", which are used to denote the currents flowing through each section, are also defined in the 
same way. When I1 is $45.6 \mathrm{~mA}$ and I2 is $12 \mathrm{~m}$, this device can oscillate at $1530 \mathrm{~nm}$, as shown in Figure 7(a). If we increase I2, another group of oscillation modes appear at $1537 \mathrm{~nm}$. The peak intensities of the two groups are equal when I 2 is $14.4 \mathrm{~mA}$. If I 2 is increased further, the $1537 \mathrm{~nm}$ group of oscillation will dominate. Similar situation occurs when I 2 is fixed and I1 is increased (Fig. 8). However, increasing I1 will increase the group of 1530nm oscillations and suppress the group of $1537 \mathrm{~nm}$ oscillations.

By collecting the two groups of oscillation modes in two detectors, the total power of each group is recorded. When I1 is fixed at $45.6 \mathrm{~mA}$ and $\mathrm{I} 2$ is increased from $12 \mathrm{~mA}$, both intensities of $1530 \mathrm{~nm}$ and $1537 \mathrm{~nm}$ oscillations will increase initially as shown in Figure 9(a). When I2 reached $14 \mathrm{~mA}$, the oscillations at $1537 \mathrm{~nm}$ begin to compete with oscillations at $1530 \mathrm{~nm}$. The intensity of $1530 \mathrm{~nm}$ oscillation begins to drop and intensity of $1537 \mathrm{~nm}$ oscillations increases sharply. Within $5 \mathrm{~mA}$ variation of $\mathrm{I} 2$, the intensity of oscillations at $1537 \mathrm{~nm}$ increased by $22 \mathrm{~dB}$, and the intensity of $1300 \mathrm{~nm}$ oscillations drop by $17 \mathrm{~dB}$ from the peak. Increasing I1 can increase the intensity $1530 \mathrm{~nm}$ oscillations by $21 \mathrm{~dB}$ and decrease the intensity of $1537 \mathrm{~nm}$ oscillations by $19 \mathrm{~dB}$ as shown in Figure $9(\mathrm{~b})$.

In the case of this device, I1 is larger than I2. Increasing I1 will increase the intensity of shorter wavelength oscillations and suppress the intensity of longer wavelength oscillations. Similar behaviors can be observed on other devices when switching is observed with large I1, I2 difference. Such as the data shown in Figure 10, I2 varies from $22.4 \mathrm{~mA}$ to $25.2 \mathrm{~mA}$, while I1 is kept at $39.2 \mathrm{~mA}$. Increasing I2 will increase the oscillation at $1527 \mathrm{~nm}$, which is of longer wavelength. When the difference between I1 and I2 is small, this role of I1 and I2 may change. In Figure 11, I1 is $48.8 \mathrm{~mA}$ while $\mathrm{I} 2$ is increased from $42.2 \mathrm{~mA}$ to $48 \mathrm{~mA}$. Increasing I 2 leads to the increase of $1499 \mathrm{~nm}$ oscillation, which is the shorter wavelength part.

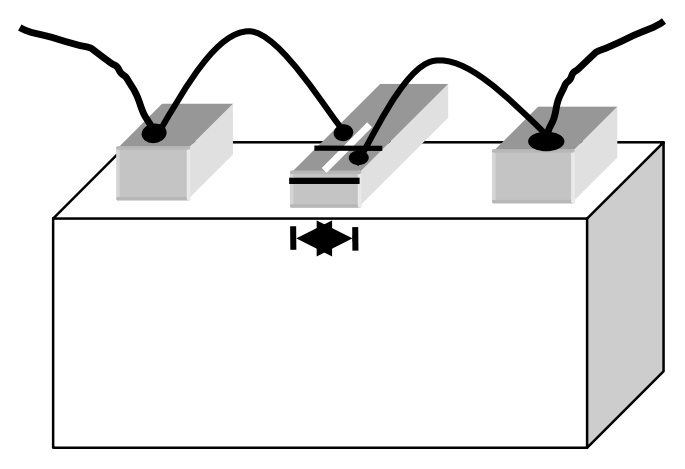

Figure 5 : The bonded device.

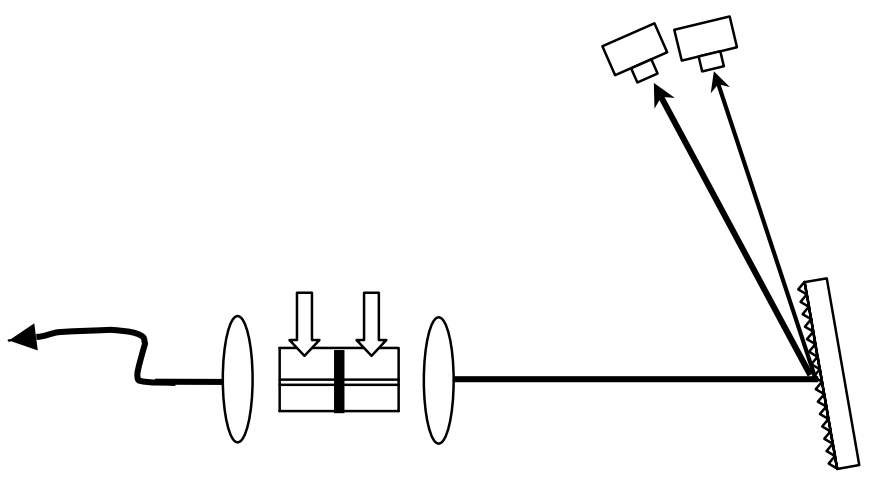

Figure 6 : The setup for power and spectrum measurement. 

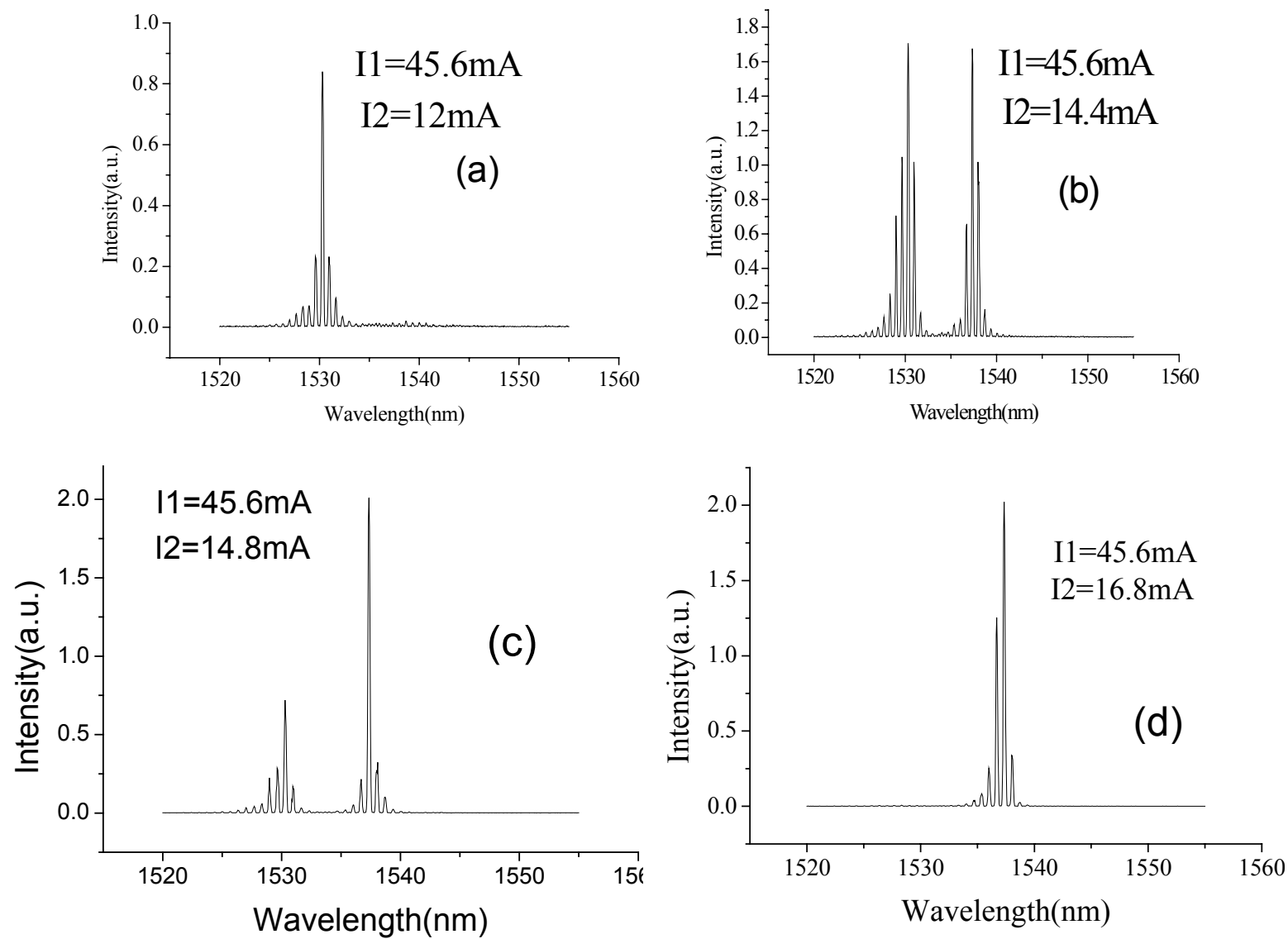

Figure 7 : The output spectrum when I1 is fixed and I 2 is increased from $12 \mathrm{~mA}$ to $16.8 \mathrm{~mA}$.
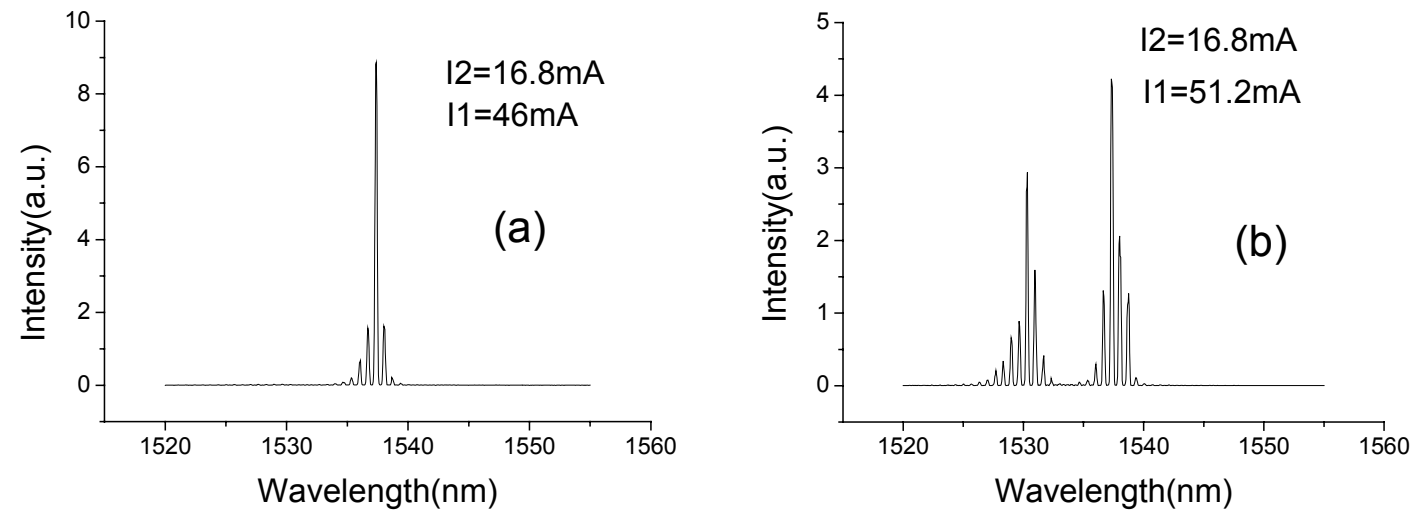

Figure 8 : The output spectrum when I1 is increased from $46 \mathrm{~mA}$ to $58.8 \mathrm{~mA}$, while I2 is fixed, (a) and (b). 

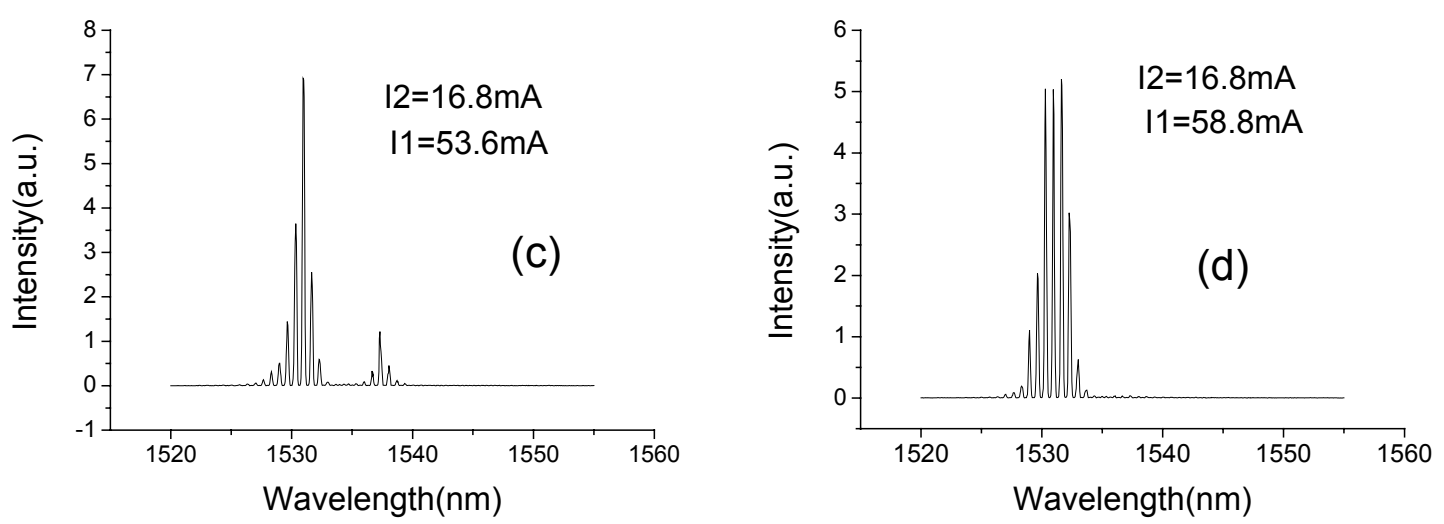

Figure 8 : The output spectrum when I1 is increased from $46 \mathrm{~mA}$ to $58.8 \mathrm{~mA}$, while I2 is fixed, (c) and (d)..

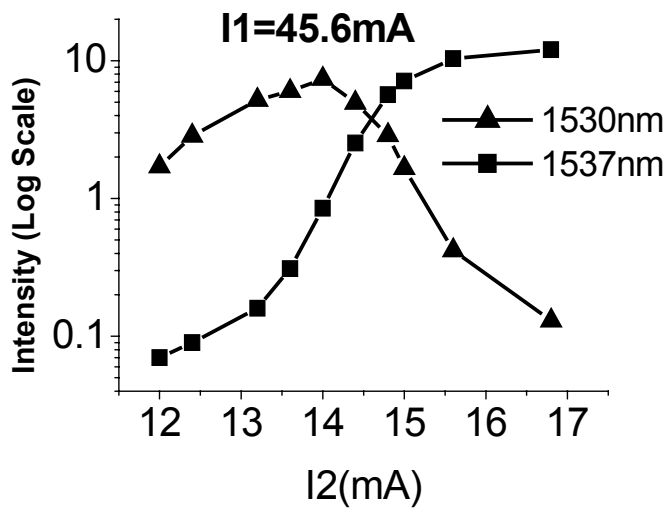

(a)I1 fixed, I2 varied

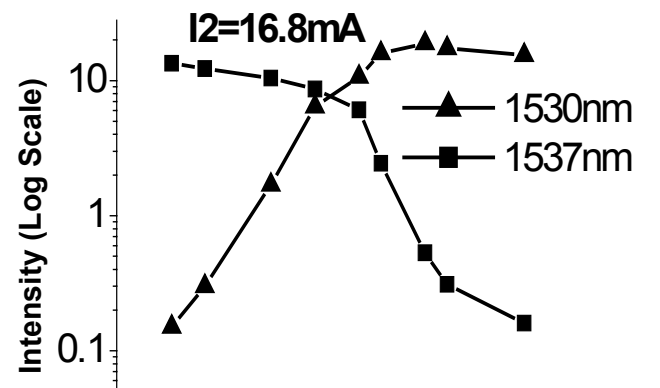

444648505254565860

$11(\mathrm{~mA})$

(b)I2 fixed, I1 varied

Figure 9: The output intensity of the two groups of oscillation modes as current is varied
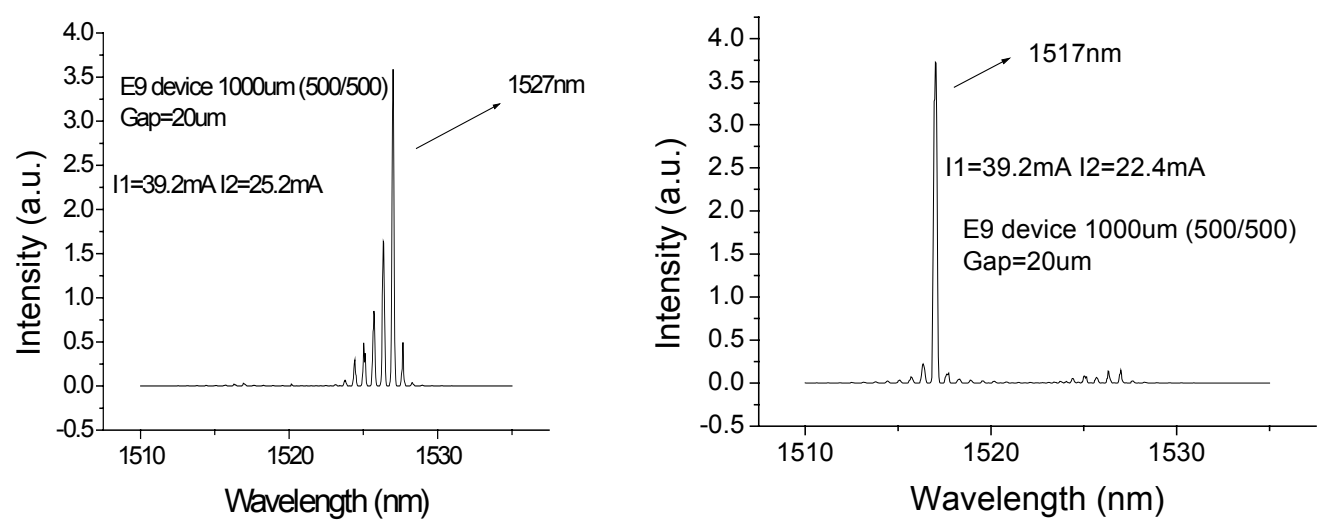

Figure 10: The variation output spectrum with current of another device, which also has large I1 I2 difference. 

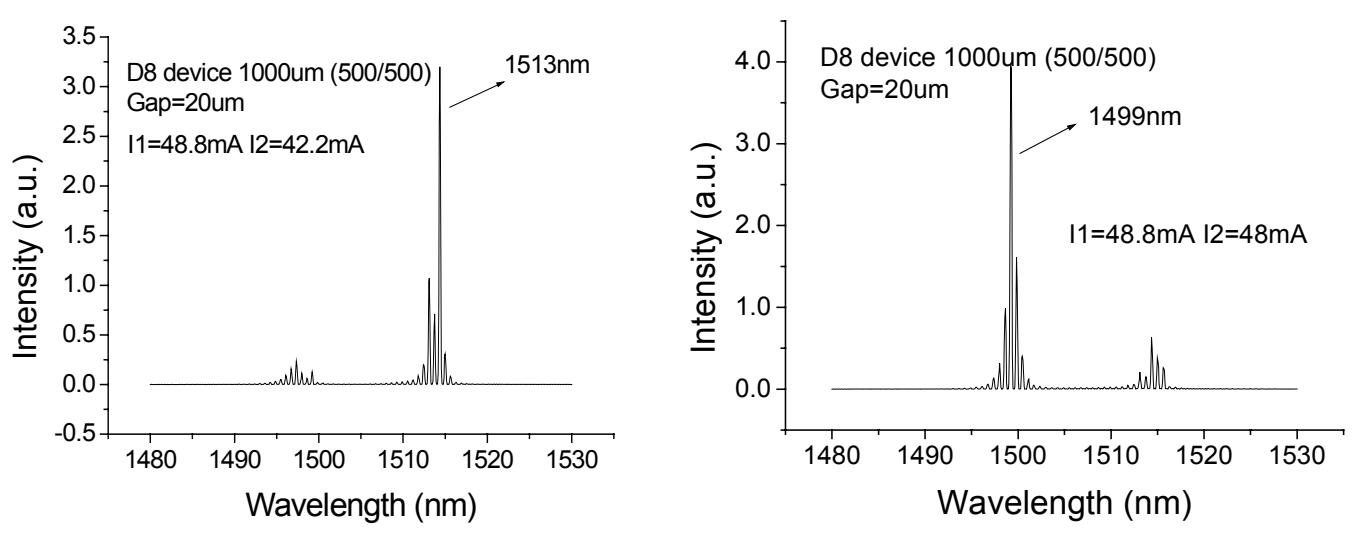

Figure 11: The variation output spectrum with current of a device, which has small I1 I2 difference.

\section{RF RESPONCE}

If these lasers are going to be used as a light source in communication network, it will be necessary to know how fast the wavelength switching can occur. Thus, we apply RF signals to these devices and measure the intensity variation of both oscillations as shown in Figure 12. The RF source is HP83732B synthesizer. An amplifier is added to increase the signals injected into the device. The two groups of laser oscillations are separated using a grating, focused to single mode fibers and fed to high-speed InGaAs detectors. The measured device is $1 \mathrm{~mm}$ in length. The two waveguide sections are $500 \mu \mathrm{m}$ each. The gap between waveguides is $20 \mu \mathrm{m}$. To prevent RF source being damaged by noise from HP8114A pulse source, the section to which RF is applied using a DC current source. The other section still uses HP8114A as current source. This device is biased at the point in which both groups of oscillations have equal output power, $0.8 \mathrm{~mW}$. The two oscillation groups are at $1514 \mathrm{~nm}$ and $1523 \mathrm{~nm}$ respectively. Figure 13 is the measured current-light transfer function at different frequency. As shown in Figure 13, the frequency response of the two-section device has a resonance at $1.5 \mathrm{GHz}$ for both wavelengths. The resonance frequency of a single section device fabricated on the same wafer and biased at $1.8 \mathrm{~mW}$ output is at $1 \mathrm{GHz}$. After the resonance frequency, they have the same $50 \mathrm{~dB} / \mathrm{dec}$ ade drop. Thus, the response of a two-section device with two laser wavelength outputs is 1.5 times faster than a single section device with only one wavelength laser output.

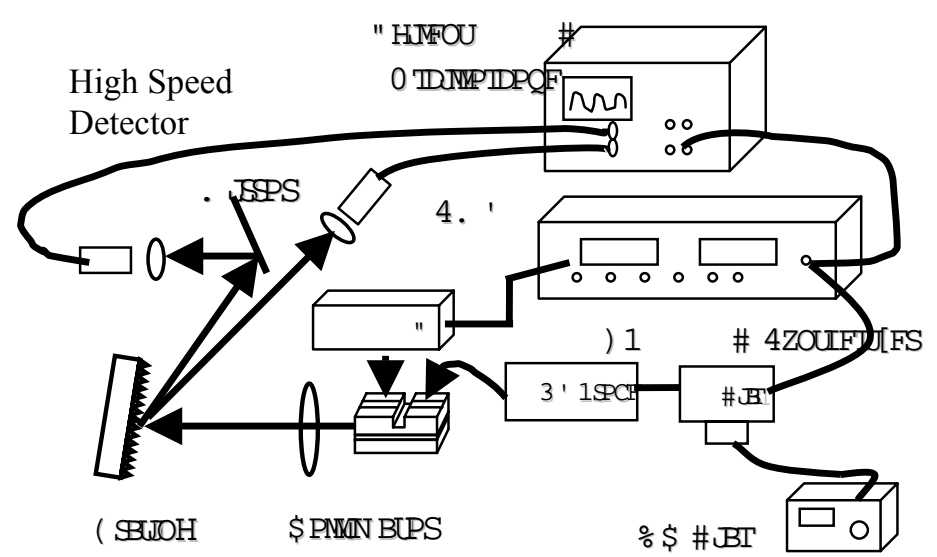

Figure 12: The setup for frequency response measurement. 


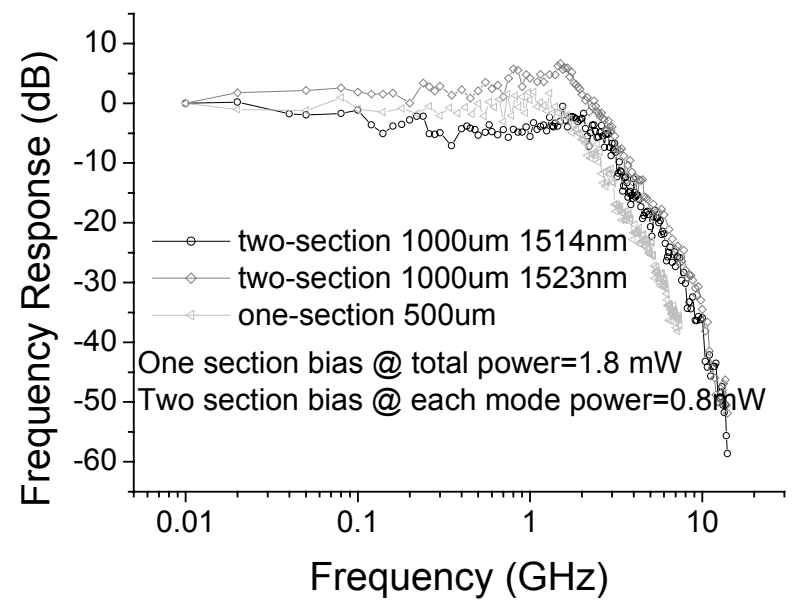

Figure 13: The current-light transfer function of two-section device and single section device.

\section{CONCLUSION}

This designed two-section laser diode is capable of providing two laser wavelengths. Laser wavelength can be modulated by direct current modulation. The output intensity at each wavelength can be varied by $20 \mathrm{~dB}$. The speed of intensity modulation of each wavelength is 1.5 times faster than the speed of a direct modulated single section laser because a part of the carriers only need to take transitions between different energy states within the active layer to retain equilibrium.

\section{REFERENCE}

1. S. Shepard, Optical Networking Crash Course, McGraw-Hill, NY., pp.120, 2001.

2. B.E.A. Saleh, M.C. Teich, Fundamentals of Photonics, Wiley, NY, pp.879,

3. Sang-Yuep Kim, Sang-Hoon Lee, Sang-Soo Lee, Jae-Seung Lee, Upgrading WDM networks using ultradense WDM channel groups, Photonics Technology Letters, IEEE, Volume: 16, Issue: 8 , Aug. 2004 Pages:1966 - 1968

4. K. Noguchi, H. Miyazawa, and O.Mitomi, “ $75-\mathrm{GHz}$ broadband Ti:LiNBO3 optical modulator with ridge waveguide." Electron. Lett. 30, 949 (1994)

5. E. L. Wooten, K. M. Kissa, A. Yi-Yan, E. J. Murphy, D. A. Lafaw, P.F. Hallemeier, D. Maack, D. V. Attanasio, D, J. Fritz, G. J. McBrien, and D. E. Bossi, "A reiew of Lithium Niobte Modulator for Fiber-Optic Commnications Systems," IEEE J. Sel. Topics Quantum Electron. 6, 69 (2000)

6. Y. Akage, K. Kawano, S. Oku, R. Iga, H. Okamoto, Y. Miyamoto and Takeuchi, “ Wide Bandwidth of over $50 \mathrm{GHz}$ Traveling-wave Electroabsorption Modulator integrated DFB lasers,” Electron. Lett. 37, pp299-300, 2001

7. S. Hansmann, H. Hillmer, H. Walter, H. Burkhard, B. Hubner, and E. Kuphal, Variation of coupling coefficients by sampled gratings in complex coupled distributed-feedback lasers,” IEEE J. Select. Topics Quantum Electron., vol. 1, pp. 341-345, June 1995.

8. Pallab Bhattacharya, Siddhartha Ghosh, Sameer Pradhan, Jasprit Singh, Member, IEEE, Zong-Kwei Wu, J. Urayama, Kyoungsik Kim, and Theodore B. Norris, Carrier Dynamics and High-Speed Modulation Properties of Tunnel Injection InGaAs-GaAs Quantum-Dot Lasers, IEEE JOURNAL OF QUANTUM ELECTRONICS, VOL. 39, NO. 8, p.952-962, AUGUST 2003

9. X. Zhang, A. Guitierrez-Aitken, R. Jambunathan, J. Singh, P. Bhattacharya, C. Caneau, and R. Bhat, "0.98 m multiple-quantum-well tunneling injection laser with $98 \mathrm{GHz}$ intrinsic modulation bandwidth," IEEE J. Select. Topics Quantum Electron., vol. 2, pp. 309-314, 1997. 